\title{
Physical Sensitivity of Silica Micro-Cantilevers Fabricated Using Direct UV Writing and Micromachining
}

\author{
Christopher HOLMES, Lewis G. CARPENTER, Helen L. ROGERS, James C. GATES and Peter G. R. SMITH
}

Optoelectronics Research Centre, University of Southampton, SO17 1BJ, UK

E-mail: chh@orc.soton.ac.uk

\begin{abstract}
A silica micro-cantilever with intrinsically defined channel waveguide containing Bragg gratings has been fabricated using a combination of direct UV writing and physical micromachining. Through optically monitoring the response of the Bragg gratings defined within the cantilever, induced stresses can be measured at multiple locations along its length permitting multiplexed physical sensitivity. The fabricated silica cantilever is $61 \mu \mathrm{m}$ wide, $41 \mu \mathrm{m}$ thick and $3 \mathrm{~mm}$ in length. It contains three Bragg gratings and can attain $\sim 70 \mathrm{~nm}$ deflection resolution.
\end{abstract}

DOI: $10.2961 / \mathrm{jlmn} .2011 .01 .0007$

Keywords: Direct UV writing, planar Bragg gratings, micro-cantilever

\section{Introduction}

Micro-cantilevers are ubiquitous in physical, chemical and biological sensing. They have received increasing interest over recent years, as a new type of miniaturised transducer that is easy-to-use, cheap and highly sensitive. Deflection readout of a micro-cantilever structure can be broadly classified into optical [1] and electronic [2-4] means [1]. Optical based sensors typically have the advantages of immunity to electromagnetic interference, the ability to be distributed over a large spatial distance before signal amplification is required and the potential to operate in flammable environments without the risk of ignition.

Optical detection can be categorised into free-space optical levering [5], inteferometry [6,7] or the use of diffraction gratings [8]. These methods of detection are typically labelled as 'free-space' as the optical wave is not containned within the cantilever structure. This usually implies the device can not be integrated upon a single compact chip and is difficult to multiplex in a distributed sensing network. The work reported in this paper considers a planar silica micro-cantilever that guides light within the cantilevers physical structure. The reported device is fabricated from a silica-on-silicon chip, which has the inherent advantage of integration.

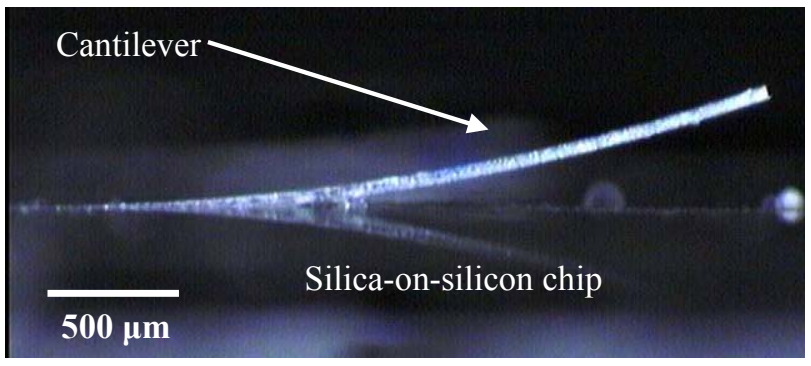

Fig. 1 Optical microscope image displaying the deflection of a silica cantilever out from the plane of a silica-on-silicon chip.

The fabricated silica micro-cantilever, pictured in Figure 1 , has a direct UV written optical waveguide and a series of multiplexed Bragg gratings defined intrinsically within it. Applying stress to the cantilever results in a physical change in the Bragg gratings. Through monitoring the reflection spectra of the Bragg gratings using a static mode regime [1] the applied stress in the cantilever can be measured and thus its deflection obtained.

\section{Design and Fabrication}

Device fabrication is uniquely achieved through a combination of direct UV writing and physical micromachining in a silica-on-silicon platform. The combination of these novel techniques allow for rapid prototyping of silica based integrated optics. Compared to alternative methods of fabrication such as photolithography, they do not require steps that can be expensive and time consuming, particularly for small device quantities.

Direct UV Writing (DUW) has been used to simultaneously fabricate waveguides and Bragg gratings. The first stage of conventional DUW is the deposition of two silica layers upon a silicon wafer, which is $\sim 1 \mathrm{~mm}$ thick and has a thick $(17 \mu \mathrm{m})$ thermally grown oxide (silica). The silica layers are deposited using a flame hydrolysis deposition (FHD) technique and subsequently consolidated at $\sim 1300^{\circ} \mathrm{C}$. The first deposited silica layer (core layer) was 7 $\mu \mathrm{m}$ thick and doped with germanium, the second silica layer was $17 \mu \mathrm{m}$ thick and had no germanium doping. The composition and thicknesses of the substrate is illustrated in Figure 2.

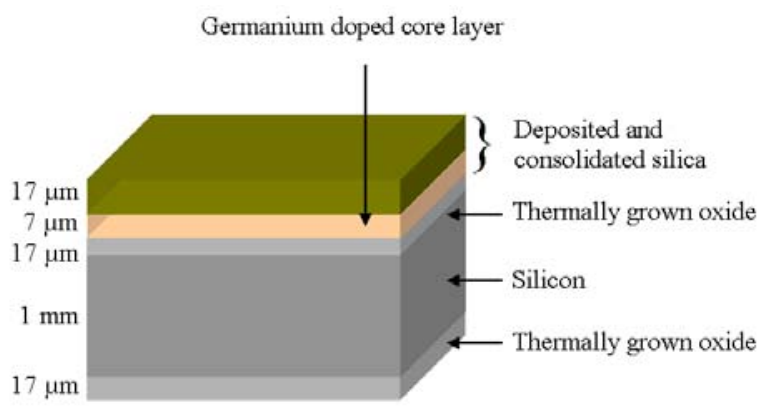

Fig. 2 Composition and thicknesses of the silica-on-silicon substrate used to fabricate the silica cantilever. 
The core layer is effectively sandwiched between two layers of silica, the thermally grown silica and the final FHD layer. This core silica layer is the only one of the three layers doped with germanium and is consequently photosensitive to UV radiation. The layer is $7 \mu \mathrm{m}$ thick to ensure vertical single mode confinement, at C-band wavelengths. In addition other dopants (boron and phosphorus) are used to control refractive index of the core and overclad layers.

The thick thermal oxide acts as a low refractive index buffer layer (underclad) for lightwaves guiding in the core layer, in addition to aiding the adhesion of the deposited silica.

The next stage of fabrication is the definition of waveguides and planar Bragg grating elements using DUW. The DUW method, used in the fabrication of the following devices, is a dual beam Direct Grating Writing (DGW) variant of DUW [9]. The established technique is ideally suited to the rapid prototyping of silica based integrated optics, requiring no photolithograph, etching or specialized cleanroom facilities.

DGW can define both waveguide and planar Bragg grating structures in a single step process. The technique focuses two coherent laser beams from an argon-ion frequency doubled $\mathrm{cw}$ laser $(244 \mathrm{~nm})$ to a $\sim 7 \mu \mathrm{m}$ diameter spot within the germanium-doped silica layer. As the beams are coherent, an interference pattern is achieved at the focal point. By traversing the sample beneath the focal point, waveguides can be directly written into the photosensitive germanium-doped silica core layer. More interestingly, by amplitude modulating the exposure during translation, planar Bragg grating structures can be written as illustrated in Figure 3. The chips in this work were fabricated in minutes, as the focused laser power used was 50 $\mathrm{mW}$ and fluences of $16 \mathrm{kJcm}^{-2}$ [9] are typically required to DUW. This is in contrast to alternative methods of fabrication such as photolithography, which for small device numbers can take days as mask designs need to be made and developed.

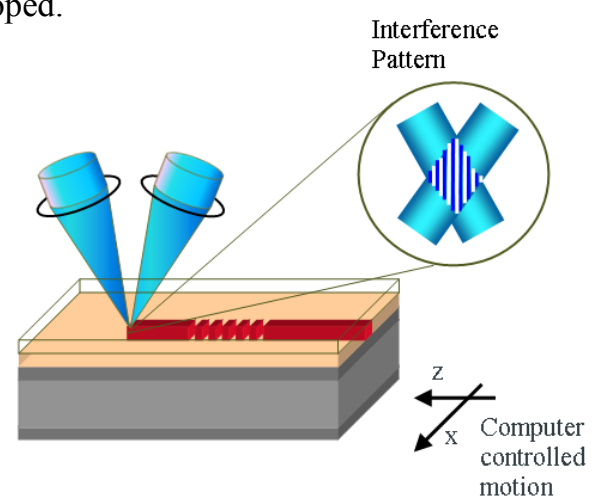

Fig. 3 Schematic of Direct UV writing process, indicating dual UV beams focused into the photosensitive germanium doped core layer to create waveguide and Bragg grating in a single fabrication step.

In the fabrication of these silica micro-cantilevers, we choose to create physical grooves in the substrate before the DGW process. The grooves were fabricated using a precision dicing saw and created the cantilever's outline into the substrate, illustrated in Figure 4 (a). The cuts were made using a $30 \mu \mathrm{m}$ wide metal bonded diamond dicing blade with an outer diameter of $54 \mathrm{~mm}$ and produced sidewalls with low surface roughness $\sim 20 \mathrm{~nm}$ Ra. The grooves were $60 \mu \mathrm{m}$ deep, such to expose the underlying silicon. Once the grooves were created UV writing was used to add the channels and Bragg gratings.
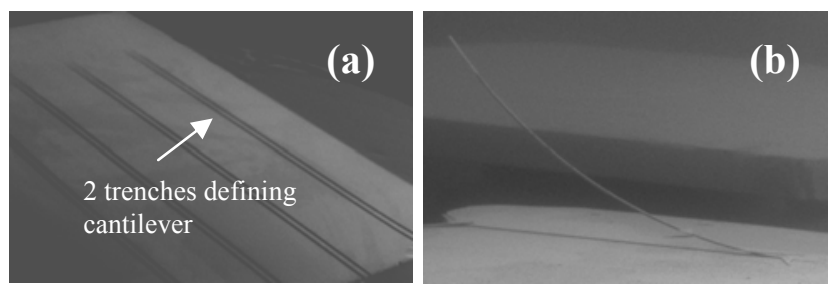

Fig. 4 Scanning Electron Microscope images of (a) diced grooves in a silica-on-silicon chip defining the outline of four cantilevers and (b) a cantilever released from the underlying silica-on-silicon chip after etching.

In the final stage of fabrication a silicon etchant $(\mathrm{KOH})$ was used to liberate the silica cantilever from the underlying silicon, as illustrated in Figure 4 (b). This etch process took 9 hours at $70^{\circ} \mathrm{C}$, with $1.0 \mathrm{M}$ of $\mathrm{KOH}$ solution. The resulting cantilever was $61 \mu \mathrm{m}$ wide and $41 \mu \mathrm{m}$ thick. The cross-sectional dimensions ensured that the evanescent field of the waveguide mode was not exposed to the surrounding medium. Thus, the spectral response of the Bragg gratings within the cantilever were only affected by induced stresses, either internal (i.e. thermal expansion) or external (i.e. applied load).

The cantilever chip reported in this paper consisted of seven single mode planar Bragg gratings, each $1 \mathrm{~mm}$ in length and placed end-to-end. Three of the Bragg gratings were located on the cantilever and four on the main body of the cantilever, as illustrated in Figure 5.

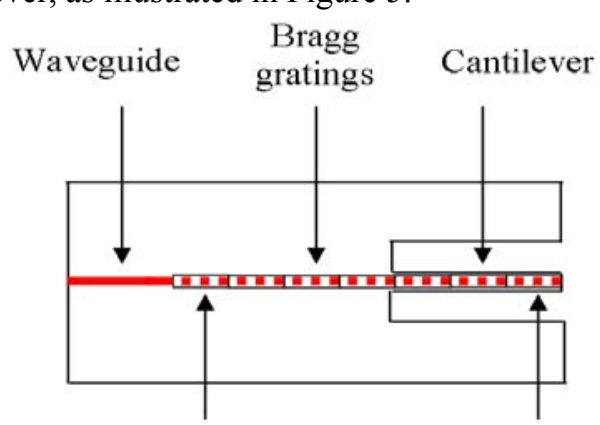

\section{Grating}

Number

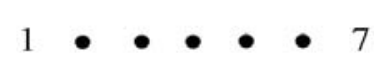

Fig. 5 Top view of cantilever chip design, indicating the physical locations of seven direct UV written Bragg gratings with respect to cantilever body.

The seven Gaussian apodised Bragg gratings were written such that they were spectrally separated by $5 \mathrm{~nm}$. The Bragg grating with the lowest central wavelength was the one physically furthest from the cantilever, i.e. grating number 1 in Figure 5.

To couple light into the device, a silicon V-groove containing a prealigned single-mode fiber was aligned to the waveguide and secured using an UV epoxy. The fiber Vgroove assembly was aligned to give optimum coupling and after attachment was mechanically robust. 
The optical spectra of the Bragg gratings were monitored in reflection using a broadband source, optical circulator and optical spectrum analyzer (OSA). The spectral resolution of the OSA was $10 \mathrm{pm}$. Through fitting the spectral Bragg reflection with a Gaussian peak, the standard error of measurement was $0.1 \mathrm{pm}$.

Figure 6 illustrates the TE reflection spectrum of the device before and after the $\mathrm{KOH}$ etching stage. The four peaks with lower central wavelengths are from gratings located on the main body of the chip. The three peaks with higher wavelengths are from gratings on the cantilever. It can be observed that whilst the gratings on the main body of the chip have shifted little, all the gratings on the cantilever have shifted to higher wavelengths after etching.

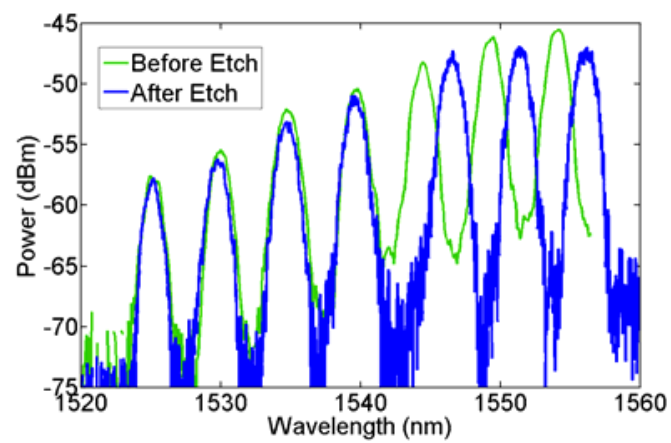

Fig. 6 Optical reflection spectrum (TE polarization) from the seven Bragg gratings before and after the cantilever is released through etching.

The large spectral shifts occurring after etching can be accounted for by considering the release of inherent stress in the silica.

\section{Thermal Stress Missmatch}

The inherent (total) stress in the silica is a combination of the thermal stress mismatch and intrinsic stress. The thermal stress mismatch can be analytically quantified through considering the silica consolidation temperatures $\left(\sim 1300^{\circ} \mathrm{C}\right)$ and the difference in expansion coefficient between silica $\left(4.9 \times 10^{-7}{ }^{\circ} \mathrm{C}^{-1}\right)$ and silicon $\left(2.6 \times 10^{-6}{ }^{\circ} \mathrm{C}^{-1}\right)[10]$. This can be expressed in terms of strain as

$$
\varepsilon=\left(\alpha_{S i O 2}-\alpha_{S i}\right) \Delta T
$$

where $\varepsilon$ is the uni-directional strain along the silica-silicon interface, $\alpha$ is the thermal expansion coefficients (subscripts indicating for silica $\mathrm{SiO}_{2}$ and silicon $\mathrm{Si}$ respectively), $T$ is the temperature difference between consolidation and operation temperature. From Equation 1 it can be calculated that the thermal strain mismatch is $\sim 2500 \mu \varepsilon$. The dopants present in the silica reduce this value [11]. However, the relative contribution is small due to the low concentrations of dopants present $(<10 \%)$.

The inherent stress relieved after etching, can be theoretically treated to infer the expected spectral change. The spectral change of a direct UV written Bragg grating to applied strain is understood to respond in a spectral shift $\lambda$ defined by [12].

$$
\frac{\Delta \lambda_{x, y}}{\lambda_{x, y}}=\varepsilon_{z}-\frac{n_{x, y}{ }^{2}}{2}\left(p_{11} \varepsilon_{x, y}+p_{12}\left(\varepsilon_{z}+\varepsilon_{y, x}\right)\right)
$$

where subscripts $x$ and $y$ are the transverse and longitudinal directions for a wave propagating along $z, p_{11}$ and $p_{12}$ are elements of the stress optic tensor, in this instance taken for silica. It must be noted that $\lambda_{x}$ and $\lambda_{y}$ represent the TE and TM polarizations respectively and thus $n_{x}$ and $n_{y}$ are the effective indices for TE and TM modes respectively. Calculating the indyced strains from the approximated thermal stress mismatch and placing them into Equation 2 results theoretically in a TE wavelength shift of $2.67 \mathrm{~nm}$. Figure 7 shows the measured central Bragg wavelengths (taken from data shown Figure 6) for the seven planar Bragg gratings before and after etching. The average wavelength shift for the cantilever gratings is $2.68 \mathrm{~nm}$, which agrees well with the theoretical prediction of $2.67 \mathrm{~nm}$. This underestimate can be accounted for by considering the additional contribution of intrinsic stress [10].

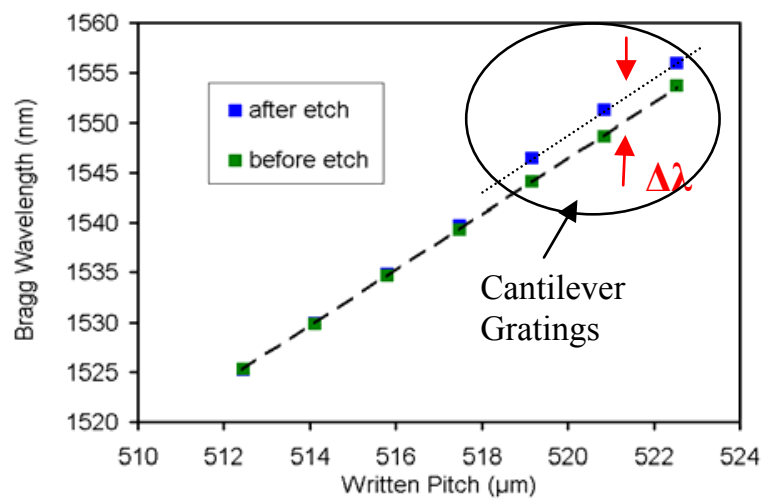

Fig. 7 The spectral shifts of the seven Bragg gratings before and after etching

Rearranging Equation 2 and assuming that $n_{x} \gg n_{y} \gg n$, the theoretical spectral change in birefringence is

$$
\Delta\left(n_{x}-n_{y}\right)=\frac{n^{3}}{2}\left[\left(p_{11}-p_{12}\right)\left(\varepsilon_{x}-\varepsilon_{y}\right)\right]
$$

where $\mathrm{n}$ is the average effective index for the TE and TM mode. The calculated theoretical birefringence shift resulting from the relief of thermal stress mismatch is $-3.30 \times 10^{-4}$. As seen in Figure 8, which shows the birefringence after etching for the seven gratings, this agrees with measured data. Grating numbers 1-4 are on the chip so they have not had their thermal stress mismatch relieved, whilst grating 5-7 are on the cantilever and show a birefringence shift of $-3.35 \times 10^{-4}$

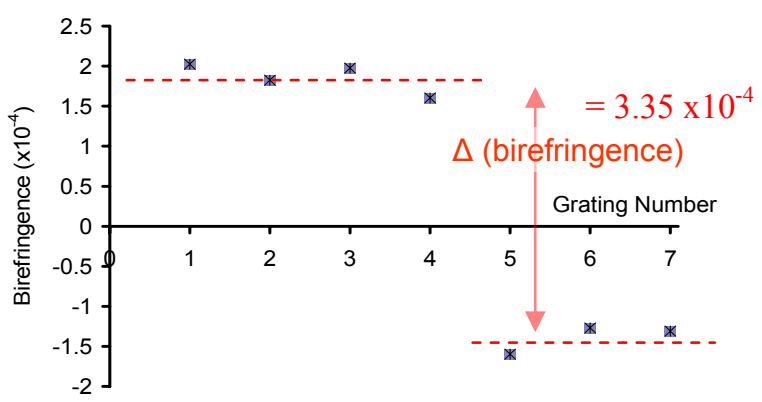

Fig. 8 The virefningence of the Bragg gratings atter etching, indicating a significant difference in birefringence for the three Bragg gratings located on the cantilever. 
So far stress monitoring has looked at the release of thermal stress mismatch as a result of fabrication. The following section shall consider stress induce deflections from externally applied load.

\section{Point Load}

The following section considers the application of a point load on the cantilever, as illustrated schematically in Figure 9.

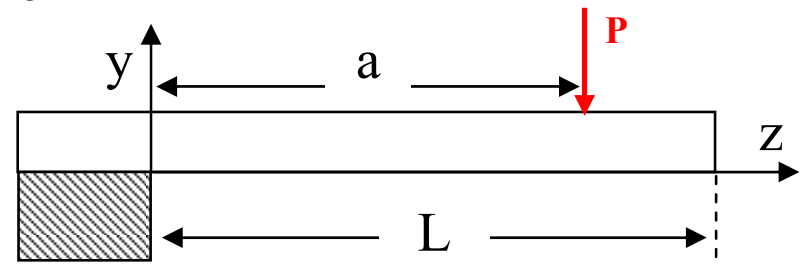

Fig. 9 A schematic indicating the notation of applied point load P

Load was applied to the cantilever using a KLA Tencor P16 stylus profiler. This can apply loads to the cantilever (up to $490 \mu \mathrm{N}$ ) and map the respective deflection (up to a range of $300 \mu \mathrm{m}$ ).

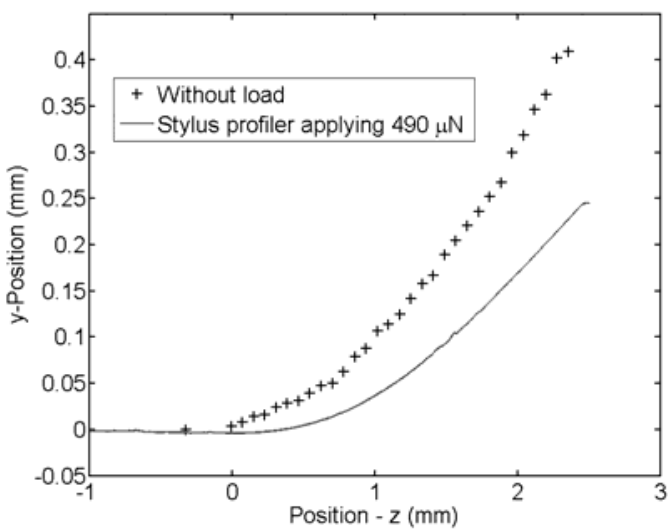

Fig. 10 The vertical displacement of the stylus profiler when scanning the top of the cantilever with an applied load of $490 \mu \mathrm{N}$

Figure 10 illustrates the cantilever's deflection under a point load of $490 \mu \mathrm{N}$ compared to the absence of load. In this measurement the stylus profiler has scanned $1 \mathrm{~mm}$ of bulk chip before scanning the cantilever (note the cantilever's root is at $\mathrm{z}=0 \mathrm{~mm}$ ). As the profiler has a vertical range of $300 \mu \mathrm{m}$ the 'without load' data was measured using a calibrated optical image. The tip deflection resulting from $490 \mu \mathrm{N}$ load is $165 \mu \mathrm{m}$.

Approximating the thermal oxide and silica layers as being homogenous and isotropic [12] and the cantilever as a slender beam undergoing small deflections [13] then an analytical solution for deflection $\Delta y$ can be inferred [14].

$$
P=-\frac{3 E I}{L^{3}} \Delta y=-k \Delta y
$$

Where $k$ is the spring constant, E is Young's modulus $(\sim 73$ $\mathrm{GPa}$ for silica) and $I$ is the moment of cross-sectional area. The theoretical spring constant for this simplified model is
$2.84 \mathrm{Nm}^{-1}$, which is comparable to the measured value of $2.97 \mathrm{Nm}^{-1}(\mathrm{k}=-\mathrm{P} / \Delta \mathrm{y})$.

Figure 11 shows the spectral shift of Bragg grating 6 (TE polarization), as a result of a $490 \mu \mathrm{N}$ point load being applied at horizontal positions along $z$.

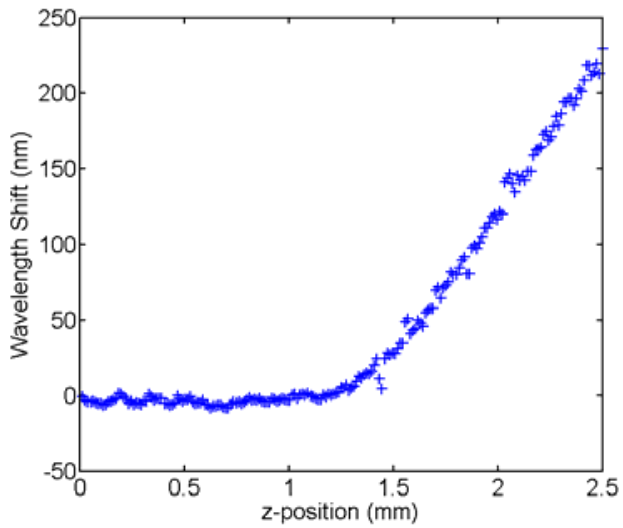

Fig. 11 The optical response of Bragg grating 6 undergoing point loads at set positions.

To understand the optical response of the grating consider the moment $M$ applied to position $\mathrm{z}$ on the cantilever, which can be expressed as [14]

$$
M(z)=\left\{\begin{array}{cc}
P a\left(1-\frac{z}{a}\right) & 0 \leq z \leq a \\
0 & a<z \leq L
\end{array}\right.
$$

Under the approximated model the moment is linearly related to strain [13]. Thus, from Equations 2 and 6 the spectral response of a grating at location $\mathrm{z}$ and undergoing an applied load at location $a$, is expected to show no spectral shift when $a \leq z$ and a linearly proportional response for $a>z$. As can be seen in Figure 11 the measured response agrees with this theory. Following this model, the greatest spectral response to applied load should be measured from grating 5. However, this is not what was observed experimentally. The reason for this discrepancy from theory is believed to be a result of the cantilever model breaking down in this area as a result of physical machining artifacts. The cantilever is physically machined using a dicing blade, meaning that near the root of the cantilever there is no abrupt detachment (as the model assumes). The cantilever outline is only fully detached from the chip at $\mathrm{z}=0.4 \mathrm{~mm}$. As a result, this section is mechanically stiffer than the other sections of the cantilever and thus the grating in this area (grating 5) is less sensitive to mechanical strain. Thus, grating number 6 has the greatest displacement sensitivity.

The maximum spectral shift for grating number 6 is $230 \mathrm{pm}$ for TM polarization. This occurs at a maximum end point deflection of $165 \mu \mathrm{m}$, meaning the total resolvable deflection is $\sim 70 \mathrm{~nm}$.

\section{Conclusions}

A silica based micro cantilever with inherently defined planar Bragg gratings has been fabricated. Using a series of multiplexed Bragg gratings the stress along the cantilever 
can be monitored. The maximum resolvable deflection of the device (in a static mode [1]) is $\sim 70 \mathrm{~nm}$.

As a result of inherent stress the fabricated cantilever deflects out of plane. Using the Bragg gratings written in the cantilever this stress can be inferred.

Using a surface profiler the optical sensitivity of the device and the cantilever's spring constant can be measured. This technique can thus be used as a calibration tool for such structures.

\section{Acknowledgments and Appendixes}

The author's thank the Engineering and Physical Science Research Council (EPSRC) for their financial support on this project.

\section{References}

[1] N.V. Lavrik, M.J. Sepaniak, P.G. Datskos, "Cantilever transducers as a platform for chemical and biological sensors“, Review of Scientific Instruments, Vol. 75 Issue 7, p2229-2253, 2004.

[2] J. Thaysen, A. Boisen, O. Hansen and S. Bouwstra, "Atomic force microscopy probe with piezoresistive read-out and a highly symmetrical Wheatstone bridge arrangement", Sens. Actuators A, Vol. 83, p47-53, 2000.

[3] M. Yang, X. Zhang, K. Vafai, and C.S. Ozkan, "High sensitivity piezoresistive cantilever design and optimization for analyte-receptor binding", J. Micromech. Microeng., Vol. 13 Issue 6, p864-872, 2003.

[4] N. Blanc, J.Brugger, N.F.de Rooij and U. Durig, "Scanning force microscopy in the dynamic mode using microfabricated capacitive sensors", J Vac. Sci. Technol. B, Vol. 14 Issue 2, p901-905, 1996.

[5] G. Meyer and N.M. Amer, "Novel optical approach to atomic force microscopy”, Appl. Phys. Lett., Vol. 53 Issue 12, p1045-1047, 1988.

[6] R. Erlandsson, G.M. McClelland, C.M. Mate and S. Chiang, "Atomic force microscopy using optical interferometry”, J. Vac. Sci. Technol. A, Vol. 6 Issue 2, p266-270, 1988.

[7] S. T. Koev, W. E. Bentley, R. Ghodssi, "Interferometric readout of multiple cantilever sensors in liquid samples" , Sens. Act. B: Chemical, Vol. 146, p245252, 2010.

[8] S.R. Manalis, S.C. Minne, A. Atalar and C.F. Quate, "Interdigital cantilevers for atomic force microscopy", Appl. Phys. Lett, Vol. 69 Issue 25, p3944-3946, 1996.

[9] G.D. Emmerson, S. P. Watts, C. B.E. Gawith, V. Albanis, M. Ibsen, R. B. Williams and P. G.R. Smith, "Fabrication of directly UV-written channel waveguides with simultaneously defined integral Bragg gratings “, Elec. Lett, Vol. 38 Issue 24, p15311532, 2002.

[10] L. Eckertova, "Physics of thin films", second ed. (Plenum, 1995).

[11] J.E. Shelby, "Introduction to glass science", second ed. (RSC 2005).

[11] C. Holmes, J.C. Gates, C.B.E. Gawith, P.G.R. Smith, "Strain tuning of a composite silica-on-silicon direct UV written planar Bragg grating”, Opt. Eng., Vol. 49 Issue 4, p044601 1-4, 2009.
[13] T. Beléndez, Cristian Neipp, A. Beléndez, 'Large and small deflections of a cantilever beam', Eur. J. Phys. Vol. 23, p371-379, 2002.

[14] R. P. Feynman, R. B. Leighton, M. Sands, The Feynman Lectures on Physics: Mainly Electromagnetism and Matter vol 2 (Reading, MA: Addison - Weseley) Chapter 38.

(Received: June 07, 2010, Accepted: January 11, 2011) 